\title{
An EXPRESS Rack Overview and support for Microgravity Research on the International Space Station (ISS)
}

\author{
Joseph J. Pelfrey ${ }^{1}$ and Lee P. Jordan ${ }^{2}$ \\ NASA Marshall Space Flight Center, Huntsville, Alabama, 35812
}

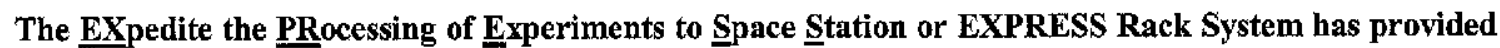
accommodations and facilitated operations for microgravity-based research payloads for over 6 years on the International Space Station (ISS). The EXPRESS Rack accepts Space Shuttle middeck type lockers and International Subrack Interface Standard (ISIS) drawers, providing a modular-type interface on the ISS. The EXPRESS Rack provides 28Vdc power, Ethernet and RS-422 data interfaces, thermal conditioning, vacuum exhaust, and Nitrogen supply for payload use. The EXPRESS Rack system also includes payload checkout capability with a flight rack or flight rack emulator prior to launch, providing a high degree of confidence in successful operations once on-orbit. In addition, EXPRESS trainer racks are provided to support crew training of both rack systems and subrack operations. Standard hardware and software interfaces provided by the EXPRESS Rack simplify the integration processes for ISS payload development. The EXPRESS Rack is designed to accommodate multidiscipline research, allowing for the independent operation of each subrack payload within a single rack. On-orbit operations began for the EXPRESS Rack Project on April 24, 2001, with one rack operating continuously to support high-priority payloads. The other on-orbit EXPRESS Racks operate based on payload need and resource availability. Over 50 multi-discipline payloads have now been supported onorbit by the EXPRESS Rack Program. Sustaining engineering, logistics, and maintenance functions are in place to maintain hardware, operations and provide software upgrades. Additional EXPRESS Racks are planned for launch prior to ISS completion in support of long-term operations and the planned transition of the U.S. Segment to a National Laboratory.

\section{Introduction}

The EXPRESS Rack serves as a platform for scientists to perform experiments on the ISS by extending resources to subrack Payload Developers (PD). The EXPRESS Rack concept was derived from the desire to have a simple hardware interface, coupled with a streamlined integration process, to get experiments to orbit quickly. The EXPRESS Rack provides standardized interfaces including Middeck Locker type interfaces and International Subrack Interface Standard (ISIS) drawer interfaces. Each EXPRESS Rack can accommodate eight single middeck lockers and two ISIS drawers. The EXPRESS Rack can also accommodate double middeck lockers and "quad" payloads that utilize the equivalent of four middeck locker spaces.

Eight EXPRESS Racks have been built, five of which have been delivered to the ISS. Four racks are configured with the Active Rack Isolation System (ARIS), two of which are currently on-orbit. The ARIS system reduces the microgravity disturbances transmitted to the rack payloads during sensitive operations. On-orbit, EXPRESS Racks are currently located in the United States Destiny Laboratory Module. Within the next year, one rack will be moved to the European Space Agency Columbus Module, and two racks will be transferred to the Japanese Experiment Module.

In addition to the EXPRESS Racks, four EXPRESS Transportation Racks (ETRs) have been built to transport EXPRESS Rack payloads to ISS. The ETR only provides a structural/mechanical interface for launch support. Payloads requiring power during ascent or descent will be transported in the Shuttle middeck. Currently, no future flights are planned for the ETR; however flight units are available if required.

The EXPRESS Rack was designed and built at NASA's Marshall Space Flight Center (MSFC). MSFC manages engineering integration for subrack payloads and sustaining engineering for all of the EXPRESS Racks. Through this integration support, compatibility of payloads within the rack and compatibility with the ISS is ensured.

\footnotetext{
${ }^{1}$ EXPRESS Rack Stage Integration Manager, NASA MSFC, VP35

${ }^{2}$ EXPRESS Rack Engineering Integration Manager, NASA MSFC, VP35
} 


\section{Hardware Provisions}

In order to support simultaneous operations of mult1-discrpline research, the EXPRESS Rack was designed to accommodate various combinations of physical hardware interfaces. The International Standard Payload Rack (ISPR), provided by the ISS program, serves as the primary structure for the EXPRESS Rack. Secondary structure is added to support the payloads and to provide mounting for the EXPRESS Rack subsystems. Subsystem hardware is located primarily in the back of the rack to maximize front access locations for the payloads, as shown in Fig. 1. Payload interfaces utilize the Shuttle Middeck Locker type interface and the ISIS drawer interface. This allows the payload positions to be utilized by multiple science disciplines and operated independently in the rack.

Middeck Locker interfaces are offered to allow payloads (a middeck locker or equivalent replacement container) that had previously flown in the shuttle middeck, Spacelab, SpaceHab, or Mir to transition to ISS using the same interface. This also allows payloads requiring power during transport or late/early access to be transferred efficiently between the Shuttle middeck and the on-orbit EXRESS Rack. Combinations of single, double or quad-locker payloads can be used for increased experiment volume.

Two 4 Panel Unit (7 inch height) ISIS drawers can be accommodated in the EXPRESS Rack. The ISIS drawer is based on the Standard Interface Rack (SIR) drawers

\section{EXPRESS 8/2 Configuration}

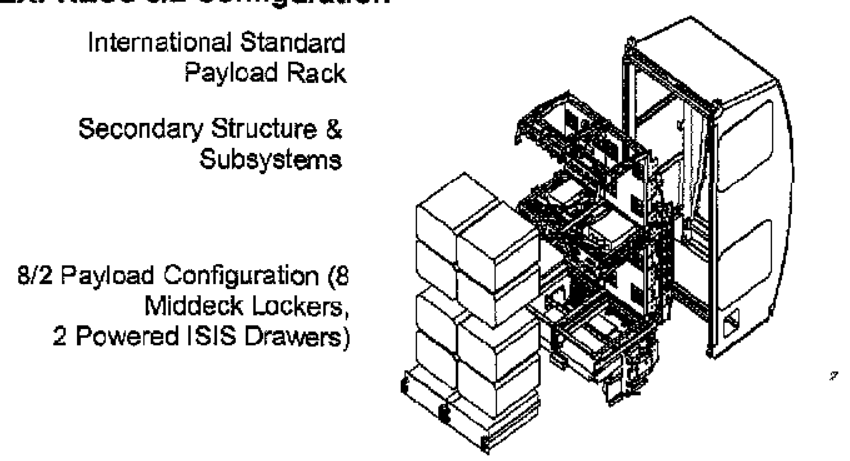

Figure 1. EXPRESS Rack Exploded View previously flown for life science payloads on Spacelab and Mir. The ISIS drawer locations can accommodate passive or powered drawer experiments. The ISIS interface uses blind-mate connectors for power and data resources provided by the rack.

The EXPRESS Rack is configured with connector panels at the top of the rack and approximately in the middle of the rack. These connector panels provide data and power interfaces for the locker positions and quick disconnects for the fluid interfaces payloads use. The EXPRESS Rack Office provides standard cables and hoses to connect payloads to the rack interface.

An EXPRESS Rack Solid State Power Controller Module (SSPCM) interfaces with the ISS provided 120Vdc power, distributes it to the other EXPRESS Rack subsystems, and converts it for distribution to the payloads. For each locker or ISIS drawer position, the EXPRESS Rack offers $28 \mathrm{Vdc}, 20$ amp maximum, configured in 5 amp increments.

Experiment control and data interfaces are provided through RS 422, Ethernet, $5 \mathrm{Vdc}$ discrete, and $+/-5 \mathrm{Vdc}$ analog interfaces. NSTC RS170A video capability is provided for each payload position. The EXPRESS Rack Interface Controller (RIC) and Payload Ethernet Hub/Bridge (PEHB) interface with the ISS data and video services to provide these interfaces to the payloads. These avionics, in conjunction with the EXPRESS Memory Unit (EMU), control the EXPRESS Rack subsystem operations, and support the communication with payloads from the ground or on-orbit. A laptop is also provided with each EXPRESS rack for crewmembers to operate and monitor EXPRESS Rack systems and payloads and can be used for specific payload experiment operations.

Thermal conditioning for payloads is provided by either air cooling or the internal water loop. Water-cooling is avarlable to payloads utulizing two ava1lable connections on each rack and is provided by the ISS moderate temperature cooling loop. The EXPRESS Avionics Air Assembly (AAA) interfaces to the moderate temperature loop and includes a water-to-air heat exchanger and fan that provides cooling air to the payload locations with 15 cfm flow. The EXPRESS Rack distributes cooling air to the interface at the rear of the lockers and drawers. It also circulates the air within the rack past the smoke detector, which is connected to the ISS Caution and Warning system. The EXPRESS Rack subsystems are primarily water-cooled through the use of ISS-developed coldplates, leaving almost all of the AAA-generated cooling air available to payloads.

The EXPRESS Rack also provides nitrogen and vacuum services. The ISS Nitrogen distribution is extended by the EXPRESS Rack to one payload quick disconnect located on the front connector panel. Likewise, the ISS Vacuum Exhaust System is extended to one location on the front of the rack that a payload can interface to via an EXPRESS-provided fluid line and quick disconnect. 
For the EXPRESS Racks with ARIS, additional subsystem hardware is included to control and monitor the ARIS portion of the rack. The ARIS components are provided by the ISS program. In addition, a Glenn Research Center provided Space Acceleration and Measurement System II (SAMS II) Remote Triaxial Sensor Electronics Enclosure (RTS-EE) is included in the EXPRESS Racks with ARIS. The RTS-EE provides a subsystem to interface to a sensor in payloads to monitor the specific microgravity enviromment in the payload.

\section{Payload Integration}

The EXPRESS Project provides direct support to the ISS Payloads Office in managing EXPRESS Rack and payload integration activities. EXPRESS payloads follow the overall ISS payload integration process which can begin as early as three years from launch. The integration cycle, as shown in Fig. 2, is supported by the EXPRESS Project at varying levels depending on payload complexity and maturity.

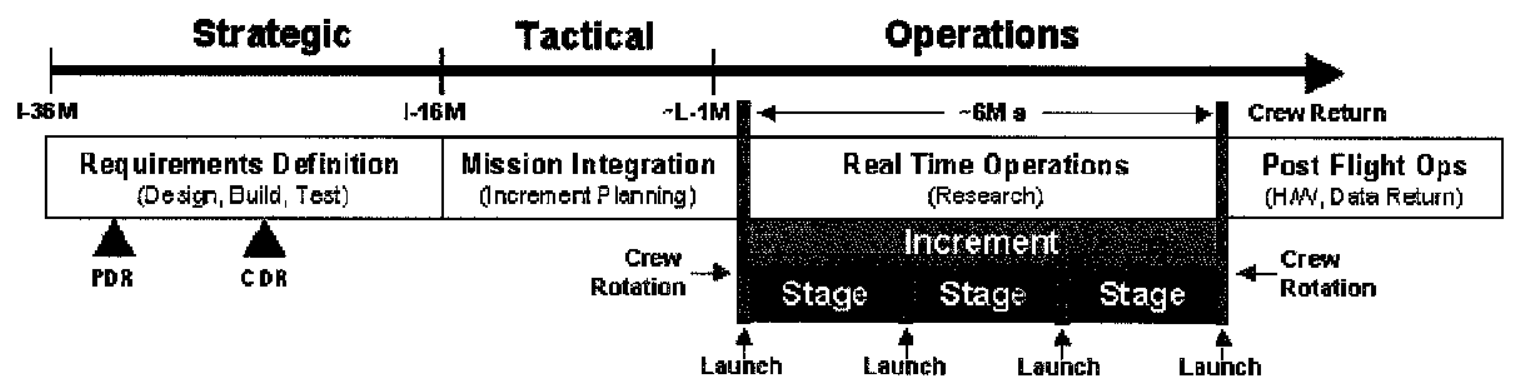

Figure 2. Payload Integration Flow Diagram

The strategic phase of integration for the payload involves the initial design and requirements definition to ensure ISS compatibility. The tactical phase begins with baseline of the research plan for a specific increment and involves activity centered on flight integration, verification and Certificate of Flight Readiness (CoFR). During the operations phase, the payload is on-orbit performing science operations with support from the real-time team. And finally, the post-flight phase involves return-to-earth, science retrieval and post-flight reports and publications.

During these phases of integration, the payload developers will coordinate with various disciplines in the ISS Payloads Office. These areas include Research Planning, Mission Integration, Engineering Integration, Safety, Test and Operations. The EXPRESS Project supports each of these discipline areas during the integration process for the rack and the subrack payloads.

\section{A. Research Planning}

EXPRESS payload users are identified through the Research Planning Working Group (RPWG) in the ISS Payloads Office. The RPWG is responsible for developing research plans for each ISS Expedition. During the research planning process, the RPWG works closely with the ISS Program Science Office and NASA Headquarters in establishing the science priorities for ISS utilization. During the utilization planning process, the EXPRESS Project provides input for payload readiness and compatibility with the research plan. Once the plan is finalized, the Payloads Office will provide direction to the project to begin implementation and tactical integration activities.

\section{B. Mission Integration}

The Mission Integration team is responsible for the overall Increment Payload Management, manifest development, integration coordination and CoFR of the payloads planned for a specific flight, stage and increment. As a service to the payload developers, a Payload Integration Manager (PIM) is assigned as a point of contact from the ISS Program to assist with the integration process. The PIM coordinates the provision of documentation and ground hardware used to checkout the payload interfaces, as well as supporting the payload developers in providing the interface information required for the ISS program. 
A Payload Integration Agreement is written to document the agreements made between the payload developer and the ISS Program, with the assistance of a Payload Integration Manager. The PIM coordinates manifest changes, tactical plan development and Certificate of Flight Readiness (CoFR) for the EXPRESS Payloads. The PIM also coordinates with the Increment Payload Managers in working issues during the tactical integration and operations phases of the payload activity.

\section{Engineering Integration}

The EXPRESS Project manages the engineering integration activity for the EXPRESS Racks and subrack payloads. Engineering integration ensures payload compatibility with the rack, the ISS modules, and transport vehicles that the payload may use. Detailed interface and requirements information is documented in the EXPRESS Rack Interface Definition Document (IDD). Based on this information and the interfaces a payload uses, an Interface Control Document (ICD) is developed for use by the integration teams. The ICD documents any deviations from the requirements and these must be agreed to by the EXPRESS Project and the ISS Program. The ICD also identifies the verification requirements by reference to the Generic Payload Verification Plan (GPVP). The GPVP outlines how each requirement is to be verified and the submittal dates for the required data.

The specific payload verification data are provided to the EXPRESS integration team and combined with the EXPRESS Rack data verify the integrated rack analytically. Each time a payload is exchanged, the new configurations are analyzed. This integrated rack information is provided to the ISS program for further element level analysis. The engineering integration process is essential to ensuring safe, successful operation of the payloads in the EXPRESS Rack.

\section{Safety}

Payloads planned for launch and operation must complete the ISS Payload Safety process to obtain approval for all aspects of payload integration and operations. Each payload is assigned a Payload Safety Engineer who assists the PD and PIM through the process. Payloads are required to complete Phase 0/1, II, III reviews depending on payload complexity. The individual payloads submit safety data packages to the Payload Safety Review Panel (PSRP) for approval. An integrated EXPRESS Rack configuration, composed of the standalone rack, payload hardware, and sample material, is submitted to the PSRP for Phase III approval. This approval is required prior to flight and/or on-orbit operations of the rack. The safety process is streamlined for payloads that have flown previously.

\section{E. Testing Support}

As part of the payload verification process, payload developers are requred to perform various tests with their hardware in order to complete its certification. Suitcase Simulators are provided by EXPRESS Rack Office as portable units to be used at a payload developer's site to support their software development and to verify data interfaces between the payload and the EXPRESS Rack. The suitcase simulator is composed of a computer with cards and software to simulate the EXPRESS Rack.

After the payload hardware is turned over to NASA, it will be integrated into a rack for integrated testing. To improve efficiency, a software simulation of the EXPRESS Rack is being developed to operate on a standard Personal Computer $(\mathrm{PC})$. This will allow payload developers to receive this software and utilize it in the development of their payload software and enable a more complete software interface development and checkout at the Payload Development site.

In order to assist payloads in their development, the EXPRESS Rack Office has established end-to-end testing capability utilizing a flight EXPRESS Rack. In the Space Systems Integration and Test Facility (SSITF) at MSFC, subrack payloads can perform commanding, telemetry downlink, fit checks, power tests and troubleshoot anomalies while connected to a flight EXPRESS Rack and simulated ISS interfaces provided by the Payload Rack Checkout Unit (PRCU). This capability also allows for personnel that will be operating the payloads on-orbit to become familiar with the payload and to validate on-orbit crew procedures. Use of this facility can be requested from the EXPRESS Rack Office and may be provided within the resources available.

\section{F. Certificate of Flight Readiness}

All payloads planning to launch, return and/or operate on ISS are required to participate in the Certificate of Flight Readiness (CoFR) process. The CoFR process is applicable to all mission phases and allows for the ISS Payloads Office to certify payload safety, integration and operational readiness. The PIM provides guidance to the payload developers throughout the CoFR process. The EXPRESS Project utilizes payload-submitted data to perform 
the integrated rack CoFR activities. Before a payload is launched, integrated or operated, all CoFR activities must be complete.

\section{EXPRESS Rack Operations}

Currently, five EXPRESS racks are onboard ISS and have all operated at some point since arriving. Since the first EXPRESS Rack was launched, at least one rack has operated continuously supporting high priority and "keepalive" science payloads. The remaining racks are scheduled to operate as resources are available to support payload requirements. With all five racks combined, EXPRESS operations have contributed to over 115,000 hours of onorbit operational time.

A Payload Rack Officer (PRO) is responsible for ground commanding during on-orbit operations of the basic EXPRESS Rack. The PRO is located at the MSFC Payload Operations Integration Center (POIC) and is responsible for configuring EXPRESS Racks for the complement of payloads on each increment. EXPRESS subsystem data is monitored by the PRO to ensure that the racks are operating nominally. The crew has the capability to control and monitor the EXPRESS Rack and its payloads on-orbit through the use of the rack laptop. Payloads are also controlled and monitored by their personnel at the POIC or at their own telescience center.

Trainer racks are provided by the EXPRESS Rack Project to support training of the crew. The trainers have the physical interfaces of the rack and simulate the operation of the EXPRESS Rack subsystems to support training for the basic operation of the rack.

Payload operations should be considered throughout the design and integration process. One of the most limiting resources on ISS is available crew time. Payloads should incorporate means to reduce crew involvement by providing for ground control, automation, and simplified interfaces required for operation.

\section{Sustaining Engineering}

The Sustaining Engineering function supports EXPRESS and derivative racks during payload integration, integrated testing, and on-orbit operations. This function includes development of software upgrades to resolve issues found in testing and to provide enhancements to allow more control from the ground. Sustaining Engineering personnel work closely with Operations personnel to troubleshoot on orbit anomalies, develop operational workarounds where possible, and to resolve any issues.

\section{Payload Users}

Through the life of the EXPRESS Rack Program, over 50 payloads have utilized EXPRESS resources while conducting ISS research. The EXPRESS Rack system allows for many areas of science and technology to utilize the ISS environment efficiently. Currently, EXPRESS Racks are supporting NASA and International Partner payloads, some of which are described below. These descriptions along with other ISS atilization information can be found at the following NASA website: http://www.nasa.gov/mission_pages/station/science/experiments/List.html

\section{A. NASA Payloads}

Advanced Astroculture TM (ADVASC) explored the benefits of using microgravity to create customized crops that withstand disease and inhospitable conditions, resist pestilence, and need less space to grow. These are qualities that will benefit space explorers and earth inhabitants. Plant growth and development in microgravity will provide a natural air and water filtration system and large-scale plant growth systems. Furthermore, ADVASC is a precursor for growing plants during extended space expeditions to the Moon and Mars.

The purpose of the Cellular Biotechnology Operations Support System (CBOSS) study was to support biotechnological research on board ISS by providing a stable environment in which to grow cells. The system was a multi-component cell incubator intended to grow three-dimensional clusters of cells in microgravity. A selfcontained apparatus, CBOSS was designed to allow multiple experiments to be performed, thereby enabling scientists to study various types of cells operating simultaneously.

Elastic Memory Composite Hinge (EMCH) studied the performance of a new type of composite hinge to determine its suitability for use in space. The experiment used elastic memory hinges to move an attached mass at one end. EMC materials tested in this experiment are stronger and lighter than current material used in space hinges and could be used in the design of future spacecraft. EMCH was performed by the crew while powered from an EXPRESS Rack and mounted to the front face to allow video to be taken during the experiment. 
EXPRESS Physics of Colloids in Space (EXPPCS) studied the kinetics of colloidal (fine particles suspended in a fluid) crystal formation and growth. These experiments provided the critical information necessary to use colloidal precursors to fabricate novel materials in the new field of colloidal engineering. Physics of Colloids in Space (PCS) focused on the growth, dynamics, and basic physical properties of four classes of colloids: binary colloidal crystals, colloid-polymer mixtures, fractal gels, and glass. These were studied using static light scattering (for size or positions of the colloids or structures formed), dynamic light scattering (to measure motions of particles or structures), flow measurement, and still imaging.

Microgravity Acceleration Measurement System (MAMS) is an ongoing study of the small forces (vibrations and accelerations) on the ISS that result from the operation of hardware, crew activit1es, as well as dockings and maneuvering. Results are used to generalize the types of vibrations affecting vibration-sensitive experiments. Investigators seek to better understand the vibration environment on the space station to enable future research. Most microgravity experiments require a quiescent environment in which the effects of gravity and other accelerations are reduced below a threshold level (determined by experiment parameters and design). Knowledge of the acceleration environment in which an experiment was operated is provided by MAMS data.

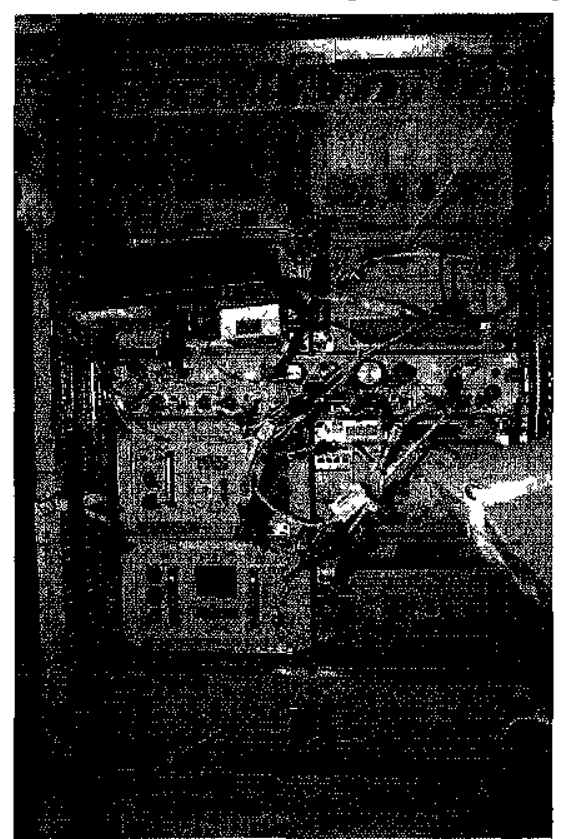

Figure 3. EXPRESS rack with payloads on ISS

The Protein Crystal Growth-Single Locker Thermal Enclosure System-Improved Diffraction Quality of Crystals (PCG-STES-IDQC) investigation was performed to obtain high quality crystals for groundbased research. Study of protein crystals is essential for visualizing proteins and developing new drugs and agricultural products. The crystals grown in microgravity are able to grow larger and more organized than those grown on Earth. Fortunately, the microgravity environment aboard the ISS is relatively free from the effects of sedimentation and convection and provides an exceptional environment for crystal growth. Crystals grown in microgravity can help scientists gain detailed knowledge of the atomic, threedimensional structure of many important protein molecules used in pharmaceutical research for cancer treatments, stroke prevention and other diseases. An EXPRESS Rack, as seen in Fig. 3, supported this experiment by providing the necessary interfaces while accommodating other payloads simultaneously.

Plant Generic Bioprocessing Apparatus (PGBA) monitored and maintained light, temperature, humidity and oxygen levels to study lignin production changes in Arabidopsis thaliana (a fast growing plant) grown in microgravity. Several different types of plants have been grown inside PGBA, including wheat, tomatoes, loblolly pine, spinach, periwinkle, white clover, pepper, sage, and purple cone flower. The ability to grow plants in space will have an enormous impact on the success of future interplanetary space exploration. Any long-term human presence on the Moon or Mars will require sustainable plant growth, which can provide a renewable food supply for explorers and assist with the maintenance of breathable air.

Space Acceleration Measurement System (SAMS-II) is an ongoing study of the small forces (vibrations and accelerations) on the ISS that result from the operation of hardware, crew activities, as well as dockings and maneuvering. Results will be used to generalize the types of vibrations affecting vibration-sensitive experiments. The residual acceleration environment of an orbiting spacecraft in low earth orbit is a complex phenomenon. Many factors, such as experiment operation, life-support systems, crew activities, aerodynamic drag, gravity gradient, rotational effects and the vehicle structural resonance frequencies (structural modes) contribute to form the overall reduced gravity environment. Weightlessness is an ideal state, which cannot be achieved in practice because of the various sources of acceleration present in an orbiting spacecraft. A complete understanding of the vibration environment will help researchers develop methods to minimize disturbances. It also allows other principal investigators to design their payloads with the vibration environment in mind.

\section{B. International Partner Payloads}

Anomalous Long Term Effects in Astronauts' Central Nervous System (ALTEA) integrates several diagnostic technologies to measure the effect of the exposure of crewmembers to cosmic radiation. It will improve the understanding of the impacts that radiation has on the human central nervous system functions, and will study the 
flashes from cosmic radiation that astronauts have reported since the Apollo flights. ALTEA will also provide an assessment of the radiation environment in the ISS. The tests conducted using the ALTEA hardware will help scientists characterize how the heavy ion radiation of space impacts the brain and whether or not that radiation causes any temporary or permanent abnormalities in the brain function and the visual system in particular.

Analyzing Interferometer for Ambient Air (ANITA) monitors 32 gaseous contaminants, including formaldehyde, ammonia and carbon monoxide, in the atmosphere on board the station. The experiment is testing the accuracy and reliability of this technology as a potential next-generation atmosphere trace-gas monitoring system for the station. This will lead to new atmospheric monitoring systems for future spaceflight.

ELaboratore Immagini TElevisive - Space 2 (ELITE-S2) will study the connection between brain, visualization and motion in the absence of gravity. ELITE-S2 will provide a system for observations on body motor control during long term exposure to microgravity and perform quantitative data collection and analysis on board the International Space Station (ISS). By recording and analyzing the three-dimensional motion of astronauts, this study will help engineers apply ergonomics into future spacecraft designs and determine the effects of weightlessness on breathing mechanisms for long-duration missions. This experiment is a cooperative effort with the Italian Space Agency, ASI. ELITE consists of an ISIS drawer, installed in an EXPRESS Rack, and 4 cameras positioned in the US Laboratory.

Molecular and Plant Physiological Analyses of the Microgravity Effects on Multigeneration Studies of Arabidopsis thaliana (Multigen) will grow Arabidopsis thaliana, a small flowering plant related to cabbage and mustard, in orbit for three generations. The results of this investigation will support future plans to grow plants on the long-duration transit to Mars. This is a cooperative investigation with the European Space Agency, ESA.

Multigen utilized the European Modular Cultivation System (EMCS) facility onboard the International Space Station (ISS). EMCS is an experiment facility for biological investigations in microgravity. For Multigen-1, seeds will mature to thale cress plants. The plant growth, including circumnutation of the stems, will be recorded using time-lapsed video in the EMCS. Once these plants reach maturity, they will be dried, stowed and returned to Earth for morphological and physiological analysis. A portion of the seeds that are produced by these plants will be used in ground-based scientific studies of morphology and physiology. The remaining seeds have been harvested and watered to observe multigenerational growth. During long-term space exploration it will be necessary to provide astronauts with regenerative sources of food as well as supplemental methods to recycle carbon dioxide into breathable oxygen. As new information about how plants grow in microgravity emerges, sustainable plant-based life support systems may be developed.

\section{ISS National Laboratory}

The National Aeronautics and Space Administration (NASA) plans to operate a share of U.S. accommodations on the International Space Station (ISS) as a national laboratory in accordance with the NASA Authorization Act of 2005. As a national laboratory, access to the ISS can be made available to other US government agencies, US private firms and academic institutions for research and development (R\&D), and industrial processing purposes. This program is intended to commence following completion of ISS assembly in late FY 2010.

As NASA plans the implementation of the ISS as a National Laboratory, the EXPRESS Rack provides simple interfaces and accommodations for payloads to utilize. Its versatility combined with proven on-orbit operations will support multiple types of payloads. The documentation and integration process is established for payloads to follow. In addition, integration efficiencies are being considered to streamline the process in support of payloads.

\section{Conclusion}

The EXPRESS Rack provides a versatile facility for use by all payload disciplines. It has also served as a basis of design for the Human Research Facility and Window Observational Research Facility racks. Designing and building the derivative racks in conjunction with the EXPRESS Racks has been a benefit to all. Some requirements have been incorporated in all the rack designs, while others have been developed uniquely for a specific configuration. Utilization of common avionics hardware in these racks has made the sustaining engineering function and the sparing of hardware more efficient. The successful on-orbit operations and versatility of the EXPRESS Rack has facilitated the operations of many scientific areas, with the promise of continued payload support for years to come. Process improvements and enhancements continue to improve the accommodations and make the integration and operations process more efficient. The shortened integration process along with the simple hardware and software interfaces will benefit Space Station researchers for many years to come. 